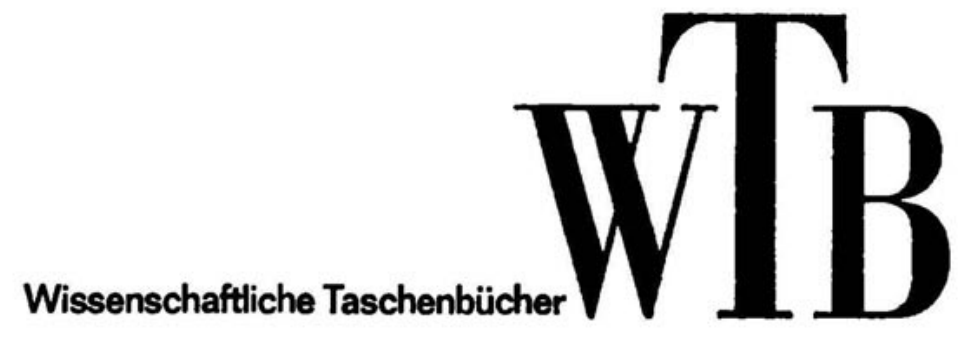

Chemie

Adolf Zschunke

Kernmagnetische

Resonanz-

spektroskopie

in der organischen Chemie

Akademie-Verlag · Berlin 


\author{
Reihe BIOLOGIE \\ HANS BERGNER \\ Tlerernāhrung
}

HeLmUt Friemel / JOSEF Brock Grundlagen der Immunologle

HELLMUTH GIBLER

Forstschutz

HEINZ GRILRR

Okologle

der Land- und SUBwassertiere

PaUl hoffunan

Photosynthese

EbERHARD HoFmanN

Elweibe und Nucleinsäuren

als biologische Makromoleküle

Dynamische Biochemie, Teil I

\section{Eberhard Hofmany}

Enzyme und energiebereitstellende

Reaktionen des Stoffwechsels

Dynamische Biochemie, Teil II

Eberhard HoFmanN

Der Stoffwechsel lebendiger Systeme und selne Regulation

Dynamische Biochemie, Teil III

Ebirhard Hotmann

Biochemle der Vererbung,

Differenzierung

und anderer Zellfunktionen

Dynamische Biochemie, Teil IV

FRIEDRICH JACOB

Bewegungsphyslologle der Ptlanzen

Gerhard Lerch

Pflanzenökologle
JoACHIM NitsGhMANN

Entwicklung bel Mensch und Tier

HANS-W ERNER NOLTE

Pflanzenschutz in der Landwirtschaft

KLAUS ODRNiNg

Parasitismus

Alfred Palissa

Bodenzoologie in Wissenschaft,

Naturhaushalt und Wirtschaft

Martin SchMidT

Pflanzenschutz im Gartenbau

ULRICH SEDLAG

Blologlsche Schädlingsbekämpfung

DIETER SPAAR

HELMUT KLEINHEMPEL

HANS JOAOHIM MULLER

Kuats naumani

Bakteriosen der Kulturpflanzen

LDDWig SPannhof

Zellen und Gewebe der Tlere

GUNTER TEMBRock

Grundlagen des Tierverhaltens

GUNTER TEMBROCK

Biokommunikation

Informationsübertragung

im biologischen Bereich

Teil I und II

Eberhard TEUSCheK

Pharmakognosie

Teil I und II

REAINe WITKOWSKI

Falko H. Herralany

Einflihrung in die kllnische Genetik

Gerald Wolp

Neuroblologle 


\section{Reihe CHEMIE}

HANS-MaRtin BarcheT

Chemie photographischer Prozesse

ULRICH BEHRENS

MANFRED RINGPFEIL

Milurobielle Polysaccharide

ROLF BORSDORF

Manfred Scholz

Spektroskoplsche Methoden

In der organischen Chemie

\section{HANS DAWOZYNSKI}

Temperaturbeständige Faserstoffe aus organischen Polymeren

HaNs DawozrNskI

Temperaturbeständige Faserstoffe aus anorganischen Polymeren

WERNGR DOPKE

Stereochemie

organischer Verbindungen

Martin Finke / WaLter LeIPNITZ Moderne Methoden der Erdölanalyse

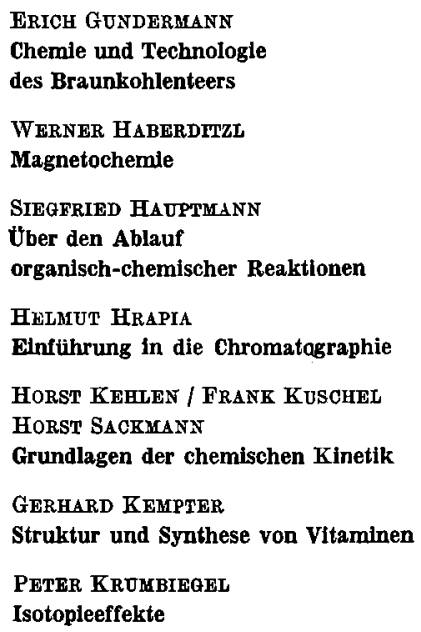

KLAUS KUHNE

Werkstoff Glas

DIETER MERKeL

Riechstoffe

DIETER ONEEN

Steroide

Burkart PhilipP

Gerhard Reinisoh

Grundlagen

der makromolekularen Chemie

JoACHIM RIEMER

Quantitative organische Miluroanalyse

ROLF SOHÖLLNER

Die Oxydation

organischer Verbindungen

mit Sauerstoff

WOLFGaNG WAGNER

Chemische Thermodynamik

GUNTHER WAGNeR

HANS KUHMSTEDT

Pharmazeutische Chemie

\section{Reihe MATHEMATIK/} PHYSIK

HANS BANDEMER

ANDreas BehLManN

WOLFHART JUNG / KLaUs KIOHTER

Optimale Versuchsplanung

Frank BeIcFet

Prophylaktische Erneuerung

von Systemen

DIFTRICH BENDER

ERnst-Egon PipPig

Einheiten, Maßsysteme, SI

WOLFRAM BRAUER

HANS-W WLDEMAR STREITWOLF

Theoretische Grundlagen

der Halbleiterphysik

SthafrIED BREHMER

Einfihrung in die Maßtheorie 
Ferdinand Cap

Einführung in die Plasmaphysik

I. Theoretische Grundlagen

II. Wellen und Instabilitäten

III. Magnetohydrodynamik

JoHN CUNNINGHaM

Vektoren

GEORG DAUTCOURT

Relativistische Astrophyslk

K. Ch. Delorarov

Relativitätstheorle und Materialismus

WERNER DUTOK

Diskrete Optimlerung

ALBert Einstein

Grundzlige der Relativitätstheorie

\section{ALBERT EINSTEIN}

Über die spezielle und die

allgemeine Relativitätstheorie

HaNneLORE FISChER

JOACHIM PIEHLRR

Modellsysteme

der Operationsforschung

ALFRED FRIESER

Mikrowellenmeßtechnik

W. L. GINSBURG

Utber Physik und Astrophysik

HERBERT GOHRING

Elementare Methoden zur Lösung von Differentialgleichungsproblemen

HERBERT GOERING

Asymptotische Methoden zur Lösung von Differentialgleichungen

Mtchaed Gösset

Angewandte Automatentheorie

Band I und II

MICHAEL GOSSEL

Wahrscheinlichkeitsautomaten

und Zufallsfolgen
E. G. GolsteIN

Konvexe Optimierung

HELMUT HeSS

Der elektrische Durchschlag in Gasen

WILHELM KäMMERER

Kybernetik - eine Einführung

auf naturwissenschaftlicher Grundlage

V. I. ITARPMAN

Nichtlineare Wellen

in dispersiven Medien

ULRich KaUsmann

ElaUs Lommatzsch

FRANTIŠEK NOŽIČKA

Lineare parametrische Optimierung

VOLKER KEMPE .

Theorie stochastischer Systeme

VOLKER KEMPE

Analyse stochastischer Systeme

Teil I

A. R. KESSEL

Akustische Kernresonanz

HEINRICH KINDLER

Der Regelkrels

Dieter KLAUA

Elementare Axlome der Mengenlehre

Einführung in die

Allgemeine Mengenlehre I

DIETER KLAUA

Grundbegriffe

der axiomatischen Mengenlehre

Teil 1 und 2

Einführung in die

Allgemeine Mengenlehre II

DIETER KLAUA

Kardinal- und Ordinalzahlen

Teil 1 und 2

Einführung in die

Aligemeine Mengenlehre III 
Dieter König / Dietrich Stoyan Methoden der Bedicnungstheorie

KONRAD KREHER

Festkörperphysik

DIETER KRESS

Theoretische Grundlagen der

Signal- und Informationsübertragung

JaAn Kuntzmann

Unendliche Relhen

Jean KonTzManN

Systeme von Differentialgleichungen

JeAN KUnTzMana

Komplexe Veränderliche

DifRCK-EKkEHARD LIEBSCheir

Relativitätstheorie

mit Zirkel und Lineal

Wolfana Mramina

Kernphysikalische Elektronik

Peter Padfler / Dieter LeUsohner Kristallographische Grundbegriffe der Festkörperphysik

Harry PaUL

Lasertheorie

Teil I und II

HARRY PaUL

Nichtlineare Optik

Teil I und II

Wolfgang Pauli

Vierpoltheorie und ihre Anwendung auf elektronische Schaltungen

HaRRy Pfeifer

Theorie linearer Bauelemente

Elektronik für den Physiker I

Harky Pfetfer

Die Elektronenröhre

Elektronik für den Physiker II

HARRY PFEIFER

Schaltungen mit Elektronenröhren Elcktronik für den Physiker III
HARRY PFEIFHR

Leitungen und Antennen

Elektronik für den Physiker IV

HARRY PFEIFER

Mikrowellenelektronlk

Elektronik für den Physiker V

HaRRy PFeLFer

Halbleiterelektronik

Elektronik für den Physiker VI

Harky Pfeifer / W. Heing

Schaltungen mit Transistoren

Elektronik für den Physiker VII

ULRICH RŐSEBERG

Determinismus und Physik

J. A. Rosanow

Wahrscheinlichkeitstheorle

WOLFGANG SCHÄFER

Theoretische Grundlagen

der Stabilität technischer Systeme

ERNST SCHMOTZER

Symmetrien und Erhaltungssätze der Physlk

\section{S. SHELUDEW}

Elektrische Kristalle

N. D. SHewaNdRow

Die Polarisation des Lichtes

L. A. SKORNJAKOW

Elemente der Verbandstheorie
A. A. SMIRNow
Metallphysik

A. S. SoNIN / B. A. STRoKow
Einführung in die Ferroelektrizität

Hans-Jutrgen Treder

Elementare Kosmologie

J. F. VINSON

Optische Kohärenz

In der klassischen Theorie und

in der Quantentheorle 


\section{TEXTE UND STUDIEN}

D. M. BRINK

Kernkräfte

STEPHEN G. BRUSH

Kinetische Theorie

Teil I und II

Festkörperphysik

Entwicklungstendenzen und

Anwendungsmöglichkeiten

D. TER HAAR

Quantentheorie

W. R. HindmaRSH

Atomspektren

J. H. SANDERS

Die Lichtgeschwindigkeit

Die Schöpfer der physlkalischen Optik

Eine Artikelsammlung

GUSTAV E. R. SOBULZE

Beiträge zur Entwicklung

der Metallphysik
R. A. R. TRICKER

Die Beiträge

von Faraday und Maxwell

zur Elektrodynamik

R. A. R. TRIORER

Frühe Elektrodynamik

Vorschau auf die nächsten Bände:

W. S. LETOOHOW

Laserspektroskopie

HANS LUPPA

Grundlagen der Histochemie

Teil I und II

E. M. SAWIZKI

Perspektiven der Metallforsehung

VOLKMAR SOHURIOHT

Kernexplosionen fïr Iriedliche Zwecke

Gerhard WUNSOH

Zellulare Systeme 